\title{
The most massive pulsating white dwarf stars
}

\author{
Barbara G. Castanheira ${ }^{1}$ and S. O. Kepler ${ }^{2}$ \\ ${ }^{1}$ Department of Astronomy and McDonald Observatory, University of Texas \\ Austin, TX 78712, USA \\ email: barbara@astro.as.utexas.edu \\ ${ }^{2}$ Instituto de Física, Universidade Federal do Rio Grande do Sul \\ 91501-970 Porto Alegre, RS, Brazil \\ email: kepler@if.ufrgs.br
}

\begin{abstract}
Massive pulsating white dwarf stars are extremely rare, because of their small size and because they are the final product of high-mass stars, which are less common. Because of their intrinsic smaller size, they are fainter than the normal size white dwarf stars. The motivation to look for this type of stars is to be able to study in detail their internal structure and also derive generic properties for the sub-class of variables, the massive ZZ Ceti stars. Our goal is to investigate whether the internal structures of these stars differ from the lower-mass ones, which in turn could have been resultant from the previous evolutionary stages.

In this paper, we present the ensemble seismological analysis of the known massive pulsating white dwarf stars. Some of these pulsating stars might have substantial crystallized cores, which would allow us to probe solid physics in extreme conditions.
\end{abstract}

Keywords. stars: oscillations (including pulsations), stars: white dwarfs

\section{Introduction}

According to the best current evolutionary models, all single stars with masses below 9-10 $M_{\odot}$ will end up their lives as white dwarf stars. The final product contains important information about their previous phases, which might be difficult to model due to some more complicated physical processes, such as nuclear reactions and mass loss. As white dwarf stars are basically just cooling, their structure is the simplest in comparison to all previous phases.

The white dwarf interior is a Coulomb plasma of ions and degenerate electron gas, which at lower temperatures will crystallize. The crystallization process emits latent heat, delaying cooling (Winget at al. 2009). For massive white dwarf stars, because of their smaller radii, crystallization is expected to start at higher temperature.

As white dwarf stars cool down, they cross four distinct instability strips (Castanheira et al. 2010, Nitta et al. 2009, Montgomery et al. 2008, Werner \& Herwig 2006), depending on their temperature and atmospheric composition. The ZZ Ceti stars (or DAVs) are stars with atmospheres dominated by hydrogen. They are observed to pulsate with a few independent modes in a narrow temperature range of about $2000 \mathrm{~K}$ (e.g. Bergeron et al. 2004, Mukadam et al. 2004). Within the instability strip, as the ZZ Ceti stars cool, the pulsation modes change both in amplitude (energy) and period (thermal timescale). Close to the blue edge of the strip, when the stars start to pulsate, we observe lowamplitude (few mma) short-period $(\sim 200 \mathrm{~s})$ modes, while close to the red edge, the mode periods are much longer (up to $\sim 1500 \mathrm{~s}$ ) and amplitudes are higher (several mma). The position of the instability strip also depends on mass (Giovannini et al. 1998, Hermes et al. 2012): the higher the mass, the hotter the stars that will start to pulsate. 
All these effects have been well studied for the stars with masses close to the main value of the mass distribution, around $0.6 M_{\odot}$. With the recent discoveries of the extreme low-mass white dwarf stars (Hermes et al. 2012), these dependencies are starting to be investigated for the lower-mass end. Our motivation is to continue the work started by Castanheira et al. (2013) and study the high-mass pulsating white dwarf stars. Our ultimate goal is to be able to constrain their internal structure and determine the differences and similarities between high- and normal-mass white dwarf stars.

One of the most exciting reasons to study massive white dwarf stars is that they are potential precursors of SN Ia. These SN explosions occur when a nearby companion fills its Roche lobe and transfers mass to the white dwarf, which then exceeds the Chandrasekhar mass limit, or by the merging of two white dwarf stars. The luminosity of SN Ia, when used as standard candles, led to the important important discovery of the accelerated expansion of the Universe (Riess et al. 1998, Perlmutter et al. 1999).

\section{Properties of the massive pulsating white dwarf stars}

Because of the intrinsically small number of pulsation modes, we have performed a seismological study of massive white dwarf stars using their ensemble properties. We made the assumption that, if similar sets of modes are observable in several stars, their internal structure should be similar. The larger number of massive pulsators allows us to probe the ensemble internal structure of the high-mass end of the ZZ Ceti instability strip. The results from our study can even be extended to the non-variable hydrogendominated atmosphere white dwarf stars.

To our knowledge, this is the first attempt to study the ensemble properties of massive pulsating white dwarf stars. With almost 30 stars more massive than $0.8 M_{\odot}$ now known, within the known ZZ Cetis, we have a sample large enough to explore some characteristics. We chose to study only the stars with SDSS spectra (see Table 1) in order to have a homogeneous sample in terms of atmospheric determinations, i.e., $T_{\text {eff }}$ and $\log g$ (Kleinman et al. 2004, 2013).

The current best models predict that pulsations start when the partial ionization zone of hydrogen $(\mathrm{H})$ deepens into the envelope. The base of the partial ionization zone masks the bottom of the convection zone. As the stars cool down, the depth of the convective zone increases, as well as its size. When the thermal timescale at the bottom of the convection zone reaches the timescale of the of $\mathrm{g}$ modes, pulsations are detected. Around $11500 \mathrm{~K}$, there is a sudden deepening of the convection zone in the models; the observed pulsations change in character, with more modes with long periods and high amplitudes excited. For normal-mass white dwarf stars at temperatures slightly lower than $11000 \mathrm{~K}$, the stars stop pulsating, defining the red edge of the ZZ Ceti instability strip. The observed amplitudes decrease towards the cool part of the strip, consistent with an increase of the depth of the convective zone (Brickhill 1991, Mukadam et al. 2006). These were the aspects investigated for the massive pulsating white dwarf stars.

In Fig. 1, we plot $T_{\text {eff }}$ as a function of the main observed period (largest amplitude). We can clearly see that there are two families of solutions. The best fits for these two families are, for periods smaller than $500 \mathrm{~s}$ :

$$
P=895.53[\mathrm{~s}]-0.06[\mathrm{~s} / \mathrm{K}] T_{\text {eff }}
$$

and for periods larger than $500 \mathrm{~s}$ :

$$
P=3186.54[\mathrm{~s}]-0.21[\mathrm{~s} / \mathrm{K}] T_{\text {eff }}
$$


Table 1. List of pulsating white dwarf stars with masses above $0.8 M_{\odot}$. Temperature and mass were determined from the SDSS spectra (Kleinman et al. 2004, 2013). In the last column, we give the reference regarding variability.

\begin{tabular}{|c|c|c|c|c|c|c|}
\hline & Star & $\begin{array}{l}T_{\text {eff }} \\
(\mathrm{K})\end{array}$ & $\begin{array}{l}\text { Mass } \\
\left(M_{\odot}\right)\end{array}$ & $\begin{array}{l}\text { Main period } \\
(\mathrm{s})\end{array}$ & $\begin{array}{l}\text { Amplitude } \\
(\mathrm{mma})\end{array}$ & Reference \\
\hline WD & J0000-0046 & $10831 \pm 224$ & $0.83 \pm 0.11$ & 611.42 & 23.0 & Castanheira et al. (2006) \\
\hline W D & J0048+1521 & $11260 \pm 139$ & $0.82 \pm 0.04$ & 615.3 & 24.8 & Mulally et al. (2005) \\
\hline W D & $\mathrm{J} 0111+0018$ & $11765 \pm 92$ & $0.81 \pm 0.03$ & 292.97 & 22.13 & Mukadam et al. (2004) \\
\hline WD & J0303-0808 & $11408 \pm 280$ & $0.94 \pm 0.08$ & 707 & 4.1 & Castanheira et al. (2006) \\
\hline W D & $\mathrm{J} 0349+1036$ & $11715 \pm 41$ & $0.86 \pm 0.01$ & 184.5 & 3.76 & Castanheira et al. (2013) \\
\hline W D & $\mathrm{J} 0825+4119$ & $11830 \pm 324$ & $0.92 \pm 0.08$ & 653.4 & 17.1 & Mukadam et al. (2004) \\
\hline WD & $\mathrm{J} 0855+0653$ & $11075 \pm 108$ & $0.88 \pm 0.04$ & 849.88 & 39.09 & Castanheira et al. (2006) \\
\hline WD & $\mathrm{J} 0925+0509$ & $10922 \pm 51$ & $0.86 \pm 0.02$ & 1378.93 & 5.25 & Castanheira \& Kepler (2009) \\
\hline WD & $\mathrm{J} 0940+0050$ & $10731 \pm 125$ & $0.92 \pm 0.06$ & 254.67 & 17.70 & Castanheira et al. (2013) \\
\hline WD & J1200-0251 & $11986 \pm 143$ & $0.82 \pm 0.04$ & 304.78 & 23.69 & Castanheira et al. (2013) \\
\hline WD & $\mathrm{J} 1216+0922$ & $11346 \pm 268$ & $0.81 \pm 0.08$ & 823 & 45.2 & Kepler et al. (2005) \\
\hline W D & J1222-0243 & $11451 \pm 105$ & $0.80 \pm 0.03$ & 396 & 22.0 & Kepler et al. (2005) \\
\hline WD & $\mathrm{J} 1257+0124$ & $11546 \pm 335$ & $0.85 \pm 0.10$ & 905.8 & 46.7 & Castanheira et al. (2006) \\
\hline WD & $\mathrm{J} 1323+0103$ & $11821 \pm 277$ & $0.94 \pm 0.06$ & 612.23 & 11.7 & Kepler et al. (2012) \\
\hline WD & $\mathrm{J} 1337+0104$ & $11511 \pm 407$ & $0.97 \pm 0.08$ & 715 & 10.0 & Kepler et al. (2005) \\
\hline WD & $\mathrm{J} 1612+0830$ & $12026 \pm 126$ & $0.90 \pm 0.03$ & 114.97 & 5.17 & Castanheira et al. (2013) \\
\hline WD & $\mathrm{J} 1641+3521$ & $11277 \pm 203$ & $0.84 \pm 0.09$ & 809.3 & 27.3 & Castanheira et al. (2006) \\
\hline WD & $\mathrm{J} 1650+3010$ & $11057 \pm 163$ & $1.02 \pm 0.06$ & 339.06 & 14.71 & Castanheira et al. (2006) \\
\hline WD & $\mathrm{J} 1711+6541$ & $11311 \pm 94$ & $1.02 \pm 0.03$ & 606.3 & 5.2 & Mukadam et al. (2004) \\
\hline WD & $\mathrm{J} 2128-0007$ & $11460 \pm 239$ & $0.81 \pm 0.08$ & 302.2 & 17.1 & Castanheira et al. (2006) \\
\hline WD & $\mathrm{J} 2159+1322$ & $11676 \pm 308$ & $1.05 \pm 0.07$ & 801 & 15.1 & Mulally et al. (2005) \\
\hline WD & $\mathrm{J} 2208+0654$ & $11104 \pm 29$ & $0.92 \pm 0.02$ & 757.23 & 4.46 & Castanheira et al. (2013) \\
\hline WD & J2209-0919 & $11546 \pm 184$ & $0.82 \pm 0.06$ & 894.71 & 43.94 & Castanheira \& Kepler (2009) \\
\hline WD & $\mathrm{J} 2214-0025$ & $11560 \pm 195$ & $0.83 \pm 0.05$ & 255.2 & 13.1 & Mulally et al. (2005) \\
\hline WD & $\mathrm{J} 2350-0054$ & $10394 \pm 140$ & $0.83 \pm 0.08$ & 304.3 & 17.0 & Mukadam et al. (2004) \\
\hline
\end{tabular}

One very interesting feature of our study is that the massive ZZ Ceti stars seem to avoid pulsating with the largest amplitude with periods around $500 \mathrm{~s}$, indicating a modeselection mechanism or mode trapping. Trapped modes in a compositionally stratified white dwarf model were discovered by Winget et al. (1981) for stellar masses around $0.6 M_{\odot}$.

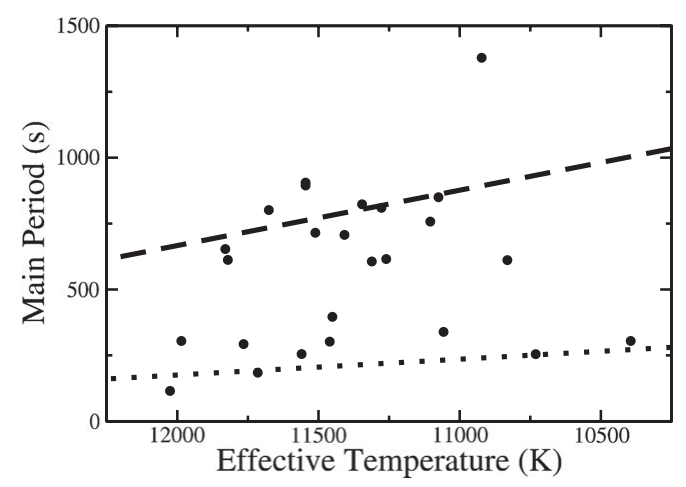

Figure 1. Main observed period as a function of effective temperature. We can identify two separate families of pulsating massive white dwarf stars.

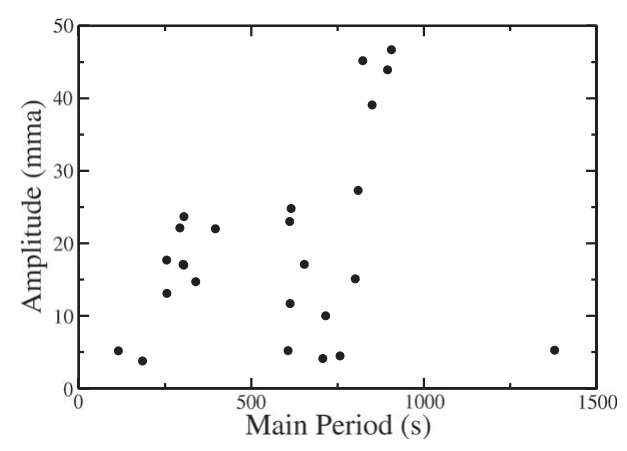

Figure 2. Observed main period versus its respective amplitude. The larger scatter for longer periods indicate that the intrinsic amplitudes are larger than for shorter pulsation periods.

In another analysis, we looked for relations between main period and observed amplitude, as plotted in Fig. 2. There is a scatter in the observed periods versus amplitude. However, we note that the scatter increases for longer periods. Because we do not know the inclination angle of the pulsation axis a priori, the observations of the changes in 
the scatter indicate that the amplitudes are intrinsically smaller for shorter periods and higher for longer periods. The fact that the longest observed period has a very small amplitude is consistent with theory: just before ceasing to pulsate, these stars should pulsate with small amplitudes and long periods.

\section{Conclusions and final remarks}

We have performed the first ensemble study of massive pulsating white dwarf stars, finding that these stars seem to avoid pulsating with a dominant mode around $500 \mathrm{~s}$. We have active observing programs at McDonald Observatory and the SOAR Telescope to look for variability in white dwarf stars. In the past years, we have focused our searches on stars that have spectroscopic masses above $0.8 M_{\odot}$. Our goal is to increase the sample of these rare pulsators to further study their ensemble and individual properties.

\section{References}

Bergeron, P., Fontaine, G., Billères, M., Boudreault, S., \& Green, E. M. 2004, ApJ, 600, 404

Brickhill, A. J. 1991, MNRAS, 252, 334

Castanheira, B. G. \& Kepler, S. O. 2009, MNRAS, 396, 1709

Castanheira, B. G., Kepler, S. O., Mullally, F., et al. 2006, A\&A, 450, 227

Castanheira, B. G., Kepler, S. O., Kleinman, S. J., Nitta, A., \& Fraga, L. 2010, MNRAS, 405, 2561

Castanheira, B. G., Kepler, S. O., Kleinman, S. J., Nitta, A., \& Fraga, L. 2013, MNRAS, 430, 50

Giovannini, O., Kepler, S. O., Kanaan, A., Wood, A., Claver, C. F., \& Koester, D. 1998, Baltic Astronomy, 7, 131

Hermes, J. J., Montgomery, M. H., Winget, D. E., Brown, W. R., Kilic, M., \& Kenyon, S. J. 2012, ApJ, 750, L28

Kepler, S. O., Castanheira, B. G., Saraiva, M. F. O., et al. 2005, A\&A, 442, 629

Kepler, S. O., Pelisoli, I., Peçanha, V., et al. 2012, ApJ, 757, 177

Kleinman, S. J., Harris, H. C., Eisenstein, D. J., et al. 2004, ApJ, 607, 426

Kleinman, S. J., Kepler, S. O., Koester, D., et al. 2013, ApJS, 204, 5

Montgomery, M. H., Williams, K. A., Winget, D. E., Dufour, P., De Gennaro, S., \& Liebert, J. 2008, ApJ, 678, L51

Mukadam, A. S., Mullally, F., Nather, R. E., et al. 2004, ApJ, 607, 982

Mukadam, A. S., Montgomery, M. H., Winget, D. E., Kepler, S. O., \& Clemens, J. C. 2006, ApJ, 640, 956

Mullally, F., Thompson, S. E., Castanheira, B. G., et al. 2005, ApJ, 625, 966

Nitta, A., Kleinman, S. J., Krzesinski, J., et al. 2009, ApJ, 690, 560

Perlmutter, S., Aldering, G., Goldhaber, G., et al. 1999, ApJ, 517, 565

Riess, A. G., Filippenko, A. V., Challis, P., et al. 1998, AJ, 116, 1009

Werner, K. \& Herwig, F. 2006, PASP, 118, 183

Winget, D. E., van Horn, H. M., \& Hansen, C. J. 1981, ApJ, 245, L33

Winget, D. E., Kepler, S. O., Campos, F., et al. 2009, ApJ, 693, L6 